\title{
PHYSICS OF LOCKED MODES IN ITER: ERROR FIELD LIMITS, ROTATION FOR OBVIATION, AND MEASUREMENT OF FIELD ERRORS
}

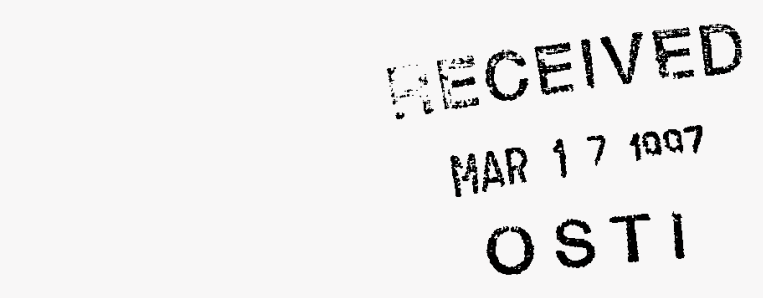

by

R.J. LA HAYE

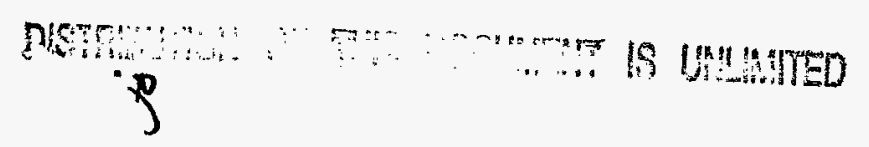

FEBRUARY 1997 
This report was prepared as an account of work sponsored by an agency of the United States Government. Neither the United States Government nor any agency thereof, nor any of their employees, makes any warranty, express or implied, or assumes any legal liability or responsibility for the accuracy, completeness, or usefulness of any information, apparatus, product, or process disclosed, or represents that its use would not infringe upon privately owned rights. Reference herein to any specific commercial product, process, or service by trade name, trademark, manufacturer, or otherwise, does not necessarily constitute or imply its endorsement, recommendation, or favoring by the United States Government or any agency thereof. The views and opinions of authors expressed herein do not necessarily state or reflect those of the United States Government or any agency thereof. 


\title{
PHYSICS OF LOCKED MODES IN ITER: ERROR FIELD LIMITS, ROTATION FOR OBVIATION, AND MEASUREMENT OF FIELD ERRORS
}

\author{
by \\ R.J. LA HAYE
}

\author{
Work supported by \\ the U.S. Department of Energy \\ under Contract No. DE-AC03-89ER51114
}

GENERAL ATOMICS PROJECT 3466

FEBRUARY 1997 


\section{DISCLAIMER}

Portions of this document may be illegible in electronic image products. Images are produced from the best available original document. 


\title{
PHYSICS OF LOCKED MODES IN ITER: ERROR FIELD LIMITS, ROTATION FOR OBVIATION, AND MEASUREMENT OF FIELD ERRORS
}

\author{
R.J. La Haye
}

\begin{abstract}
The existing theoretical and experimental basis for predicting the levels of resonant static error field at different components $m, n$ that stop plasma rotation and produce a locked mode is reviewed. (This report is a complement to Refs. 1 and 2.) For ITER ohmic discharges, the slow rotation of the very large plasma is predicted to incur a locked mode (and subsequent disastrous large magnetic islands) at a simultaneous weighted error field $\left(\Sigma_{1}^{3} w_{m 1} B_{m m 1}^{2}\right)^{1 / 2} / B_{\mathrm{T}} \gtrsim 1.9 \times 10^{-5}$. Here the weights $w_{m 1}$ are empirically determined from measurements on DIII-D to be $w_{11}=0.2, w_{21}=1.0$, and $w_{31}=0.8$ and point out the relative importance of different error field components.

This could be greatly obviated by application of counter injected neutral beams (which adds fluid flow to the natural ohmic electron drift). The addition of $5 \mathrm{MW}$ of $1 \mathrm{MeV}$ beams at $45^{\circ}$ injection would increase the error field limit by a factor of $5 ; 13 \mathrm{MW}$ would produce a factor of 10 improvement. Co-injection beams would also be effective but not as much as counter-injection as the co direction opposes the intrinsic rotation while the counter direction adds to it.

A means for measuring individual PF and TF coil total axisymmetric field error to less than 1 in 10,000 is described. This would allow alignment of coils to $\mathrm{mm}$ accuracy and with correction coils [3] make possible the very low levels of error field needed.
\end{abstract}



PHYSICS OF LOCKED MODES IN ITER: ERROR FIELD LIMITS, ROTATION FOR OBVIATION, AND MEASUREMENT OF FIELD ERRORS

\section{Contents}

Abstract $\ldots \ldots \ldots \ldots \ldots \ldots \ldots \ldots \ldots \ldots \ldots \ldots \ldots \ldots \ldots \ldots \ldots \ldots \ldots \ldots$ iii

1. Introduction $\ldots \ldots \ldots \ldots \ldots \ldots \ldots \ldots \ldots \ldots \ldots \ldots \ldots \ldots \ldots \ldots \ldots \ldots \ldots \ldots$

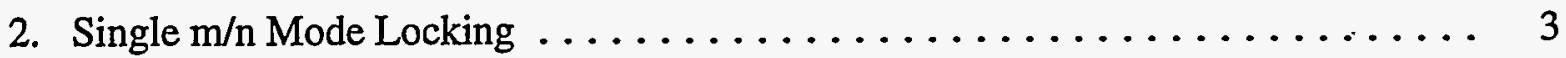

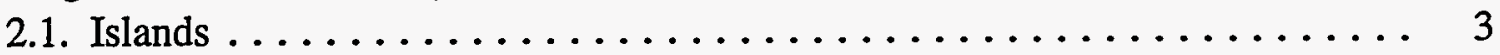

2.2. Torque/Drag on Rotation .................... 4

2.3. Single $\mathrm{m} / \mathrm{n}$ Mode, 1-D Model of Resonant Error Field Mode Locking .... . 5

3. Multimode $m / n$ Locking $\ldots \ldots \ldots \ldots \ldots \ldots \ldots \ldots \ldots \ldots \ldots$

4. ITER Multi-Mode Resonant Static Error Limits $\ldots \ldots \ldots \ldots \ldots \ldots \ldots \ldots 11$

4.1. Ohmic, Low Density . . . . . . . . . . . . . . . . . 11

4.2. Neutral Beam Rotation Drive $\ldots \ldots \ldots \ldots \ldots \ldots \ldots \ldots \ldots \ldots \ldots \ldots \ldots$

5. Error Field Measurement and Coil Alignment $\ldots \ldots \ldots \ldots \ldots \ldots \ldots \ldots 17$

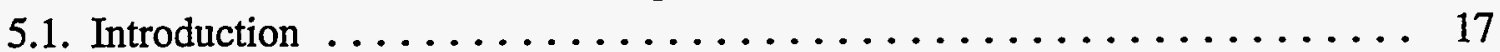

5.2. Proposed ITER Measurement and Alignment Steps . . . . . . . . . 17

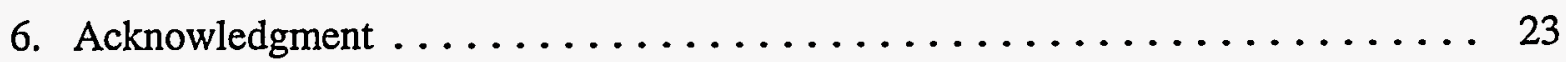

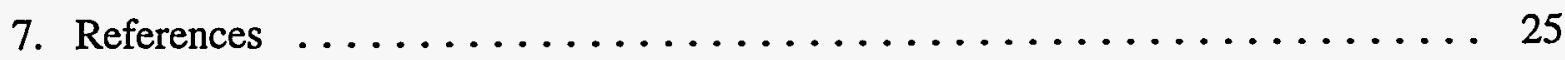

\section{Figures}

1. Model radial profiles of plasma rotation with and without error field delta function drag at singular surface $\ldots \ldots \ldots \ldots \ldots \ldots \ldots \ldots \ldots$

2. Model for relative plasma rotation and island width versus resonant static radial error field $\ldots \ldots \ldots \ldots \ldots \ldots \ldots \ldots \ldots \ldots \ldots \ldots \ldots \ldots \ldots \ldots \ldots$

3. Critical weighted relative error field versus major radius for COMPASS-C, DIII-D, and JET extrapolated to ITER $\ldots \ldots \ldots \ldots \ldots \ldots \ldots \ldots \ldots \ldots$

4. Same as Fig. 3 but calculated effect of $1 \mathrm{MeV}$ counter-injected beams

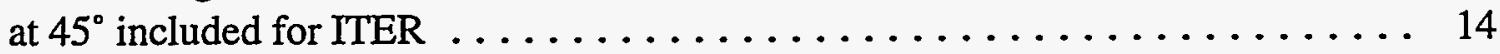

5. COMPASS-C showing permanent error field measurement assembly $\ldots \ldots \ldots 18$

6. DIII-D top view of pickup coil array $\ldots \ldots \ldots \ldots \ldots \ldots \ldots \ldots \ldots \ldots \ldots$

\section{Tables}

I. Scaling to ITER, multi-mode experiments, simultaneous $\ldots \ldots \ldots \ldots \ldots$

II. Proposed simultaneous ITER error field limits $\ldots \ldots \ldots \ldots \ldots \ldots \ldots \ldots$ 


\section{INTRODUCTION}

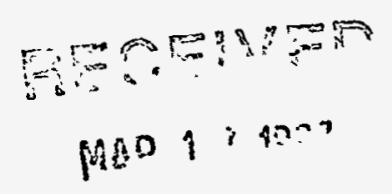

OST

Locked modes are resonant $q=m / n$ magnetic field perturbations that appear when resonant static error field exert torque/drag which stops the plasma rotation, thus locking the perturbation in the lab frame. They arise in two ways. The first way is by a spontaneous rotating Mirnov mode due to an unstable plasma configuration. This rotating magnetic perturbation cannot penetrate the resistive vacuum vessel wall, but induces a countering eddy current whose field acts on the rotating island current $J_{\theta m n}$ to exert a slowing torque. Under certain conditions, this torque can stop the rotating mode and lock it [4], a situation not of concern for this report. The second way of locking is by application of a resonant $m, n$ static error field to the plasma fluid rotating at the surface $q=m / n$. A rotating plasma is self-healing to resonant static error fields, i.e., no significant islands can be formed because the rotating singular surface acts as a conducting wall and an eddy current is induced which nearly cancels the static field, thus no island [5-8]. However, this eddy current is not exactly canceling as the singular layer has finite resistivity, thus, there is a net torque/drag which slows the rotation and can lead to locking. Once the rotation is stopped, the island cancellation is lost and an amplification of the static error field tends to occur. Thus, static error field locked modes/islands are particularly troublesome.

Rotation of ohmic plasmas is due to the electron diamagnetic drift and decreases as device size increases [9]. Thus, ITER is predicted to be very sensitive to error fields and locking. The level of tolerable error field can be significantly increased by adding rotation using co or counter injected neutral beams. Counter-injection is preferable since it adds to the electron drift. Co-injection requires extra power since it opposes the natural electron drift and must transiently drive the rotation through zero.

Very low levels of resonant static error field are possible provided: (1) careful coil design minimizes asymmetries from feeds, turn to turn transitions, etc. [10], (2) careful coil alignment is done [10], and (3) residual errors are reduced by a well designed correction coil [11]. Total $n=1$ relative errors from individual coils can be measured to $<1$ in $10^{4}[7,12]$ which translates to $\mathrm{mm}$ alignment accuracy in ITER.

In Section 2, the single mode one-dimensional (1-D) model for static error field locking and scaling with parameters is presented. Section 3 develops work on multimode models. The multimode error field limits for ITER are estimated in Section 4.1 for ohmic plasmas and in Section 4.2 for neutral beam rotation driven L-mode plasmas. Finally, a means of measuring (and aligning) PF coil and TF coil error fields is proposed in Section 5. 



\section{SINGLE $m / n$ MODE LOCKING}

\subsection{ISLANDS}

The singular surface $q=m / n$ is the analogy of a thin conducting wall of width $\delta$, radius $r$, conductivity $\sigma$, rotating at angular frequency $\omega$. The radial $B_{r m n}$ resonant [left, if left-handed plasma of form $\cos (n \phi-m \theta)$ ] static error field cannot penetrate such a rotating layer if $\omega \tau_{\text {rec }} \gg 1$ where $\tau_{\text {rec }}$ is the reconnection time (related to the plasma resistivity). Essentially, an eddy current is induced which if $\omega \tau_{\text {rec }} \rightarrow \infty$, cancels the applied $B_{r m n}$, i.e., opposes reconnection.

The full radial width of the resulting island is

$$
w_{m n}=\left(\frac{16 r_{m n} R B_{r m n}}{n s B_{\mathrm{T}}}\right)^{1 / 2}\left(\frac{2 m}{-\Delta^{\prime} r_{m n}}\right)^{1 / 2}\left[\frac{1}{1+\left(\omega \tau_{\mathrm{rec}}\right)^{2}}\right]^{1 / 4} .
$$

The first term is the "vacuum island width" [13], and is almost always inappropriate for a tokamak as it neglects both the second and third terms. The second term is the usually large amplification (neglecting rotation) due to the plasma helical current response with $\Delta^{\prime}$ the logarithmic jump in poloidal flux across the tearing layer. For broad current profiles and/or high $m, \Delta^{\prime} r_{m n} / 2 m \approx-1$ and for peaked current profiles and low $m, \Delta^{\prime} r_{m n} / 2 m \approx 0$ [14]. The third term is the rotation opposition to reconnection and can be very small if $\omega \tau_{\text {rec }}$ is large [7]. In ITER, with $m / n=2 / 1, r_{m n} \approx 0.7 a \approx 2.0 \mathrm{~m}, R=8.0 \mathrm{~m}$, shear $s=1$, $B_{r m n} / B_{\mathrm{T}} \approx 2 \times 10^{-5},-\Delta^{\prime} r_{21} / 2 m \approx 0.25$, and $\omega \tau_{\text {rec }} \approx 2 \pi \times 130 \mathrm{~Hz} \times 0.25 \mathrm{sec} \approx 200$, a vacuum island of $7 \mathrm{~cm}$ would be $14 \mathrm{~cm}(w / r=0.07)$ when locked $(\omega=0)$ and only $1 \mathrm{~cm}$ $(w / r=0.005)$ when rotating. Thus, $w_{m n} / w_{\text {vac }} \ll 1$ for rotating (also called slipping) with $\left(\omega \tau_{\text {rec }}\right)^{2} \gg 1$ and $w / w_{\text {vac }}>1$ for locked with $\left(\omega \tau_{\text {rec }}\right)^{2}<1$. Note that Hurricane and Jensen in numerical resistive viscous modeling of islands found that no island exists with slipping, not even a greatly reduced one [8].

The skin layer physics for $\tau_{\text {rec }}$ is not yet determined. The analogy is to a conducting shell wall time $\tau_{\mathrm{w}}=\mu_{0} \sigma r_{m n} \delta / 2$. Fitzpatrick suggested using the tearing layer thickness for $\delta$ so that $\tau_{\text {rec }} \approx \tau_{\mathrm{R}}^{3 / 5} \tau_{\mathrm{A}}^{2 / 5}$ or $\tau_{\mathrm{R}}^{5 / 6} \tau_{\mathrm{A}}^{1 / 3} / \tau_{\mathrm{v}}^{1 / 6}$ with viscosity where the resistive time $\tau_{\mathrm{R}}=\mu_{0} \sigma r_{m n}^{2}$, the Alfvern time $\tau_{\mathrm{A}}=R / B_{\mathrm{T}}\left(\mu_{0} \bar{n} m_{\mathrm{i}}\right)^{-1 / 2}$, and the viscous time is $\tau_{\mathrm{v}}=r_{m n}^{2} / v_{\perp}$ where $v_{\perp}$ is the perpendicular viscosity [7]. Jensen et al. suggested using the selfconsistent island width $w_{m n}$ for $\delta$, thus, $\tau_{\text {rec }} \approx \tau_{\mathrm{R}}\left(w_{m n} / r_{m n}\right) / 2 m$ [5]. In a later work, Jensen et al. suggested better agreement would be found with experiment using a self- 
consistently turbulence enhanced $\eta$ in the singular layer above that due to Spitzer collisions [15]. Wang and Bhattacharjee arrived at yet a different relation by dealing with nonconstant $\psi$ islands instead of the usual constant $\psi$ approximation used by previous authors [16].

\subsection{TORQUE/DRAG ON ROTATION}

The induced eddy current at the singular layer does not completely cancel the applied $B_{r m n}$ as the finite $\tau_{\text {rec }}$ produces a small phase shift. Thus there is a net $\left\langle J_{\theta} \times B_{r}\right\rangle_{m n}$ torque which opposes the rotation. This is given as a delta function at $q=m / n$ for $\left(\omega \tau_{\text {rec }}\right)^{2} \gg 1$

$$
T_{\phi m n}=-R \times\left(\frac{B_{r m n}^{2}}{\mu_{0}}\right)\left(\frac{r_{m n}}{q R}\right)\left(2 \pi R 2 \pi r_{m n}\right)\left(\frac{1}{\omega \tau_{\mathrm{rec}}}\right),
$$

where the second bracket is the projection of the helical torque in the toroidal direction and the fourth bracket shows that $T_{\phi m n} \rightarrow 0$ as $\omega \tau_{\text {rec }} \rightarrow \infty$, i.e., as the singular surface acts as a perfect conductor $[7,17]$.

The drag from the $\mathrm{m} / \mathrm{n}$ static resonant error field at the singular surface can slow the entire plasma rotation. Taking a momentum drive toroidal torque per unit volume $d T_{\phi} / d V$ and viscosity $v_{\perp}$ uniform in minor radius, the momentum balance with error field torque is

$$
0=\frac{d T_{\phi}}{d V}+\frac{n_{\mathrm{i}} m_{\mathrm{i}} v_{\perp}}{r} R \frac{d}{d r}\left(r \frac{d \mathrm{v}_{\phi}}{d r}\right)+\frac{T_{\phi m n}}{4 \pi R r_{m n}} \delta\left(r-r_{m n}\right)
$$

The boundary conditions are $\mathrm{v}_{\phi}(a)=0, \mathrm{v}_{\phi}\left(r_{m n}\right)$ continuous. The solution for toroidal rotation velocity $\mathrm{v}_{\phi}(r)$ is

$$
\begin{array}{rlrl}
\mathrm{v}_{\phi} & =\mathrm{v}_{0}\left(1-r^{2} / a^{2}\right)+\frac{2 \mathrm{v}_{0} T_{\phi m n} \ln \left(r_{m n} / a\right)}{\left(d T_{\phi} / d V\right) 2 \pi^{2} R a^{2}} \quad, & & 0 \leq r \leq r_{m n}, \\
& =\mathrm{v}_{0}\left(1-r^{2} / a^{2}\right)+\frac{2 \mathrm{v}_{0} T_{\phi m n} \ln (r / a)}{\left(d T_{\phi} / d V\right) 2 \pi^{2} R a^{2}} \quad, \quad \mathrm{r}_{m n} \leq r \leq a,
\end{array}
$$

with

$$
\mathrm{v}_{0}=\frac{\left(d T_{\phi} / d V\right) a^{2}}{4 n_{\mathrm{i}} m_{\mathrm{i}} v_{\perp} R}
$$

The solutions with and without error field drag are shown in Fig. 1. While the greatest effect is at $r=r_{m n}$, viscosity lowers rotation across the profile. The effect of singular surface torque can be readily generalized to multimodes and/or to nonuniform $d T_{\phi} / d V, v_{\perp}$. 


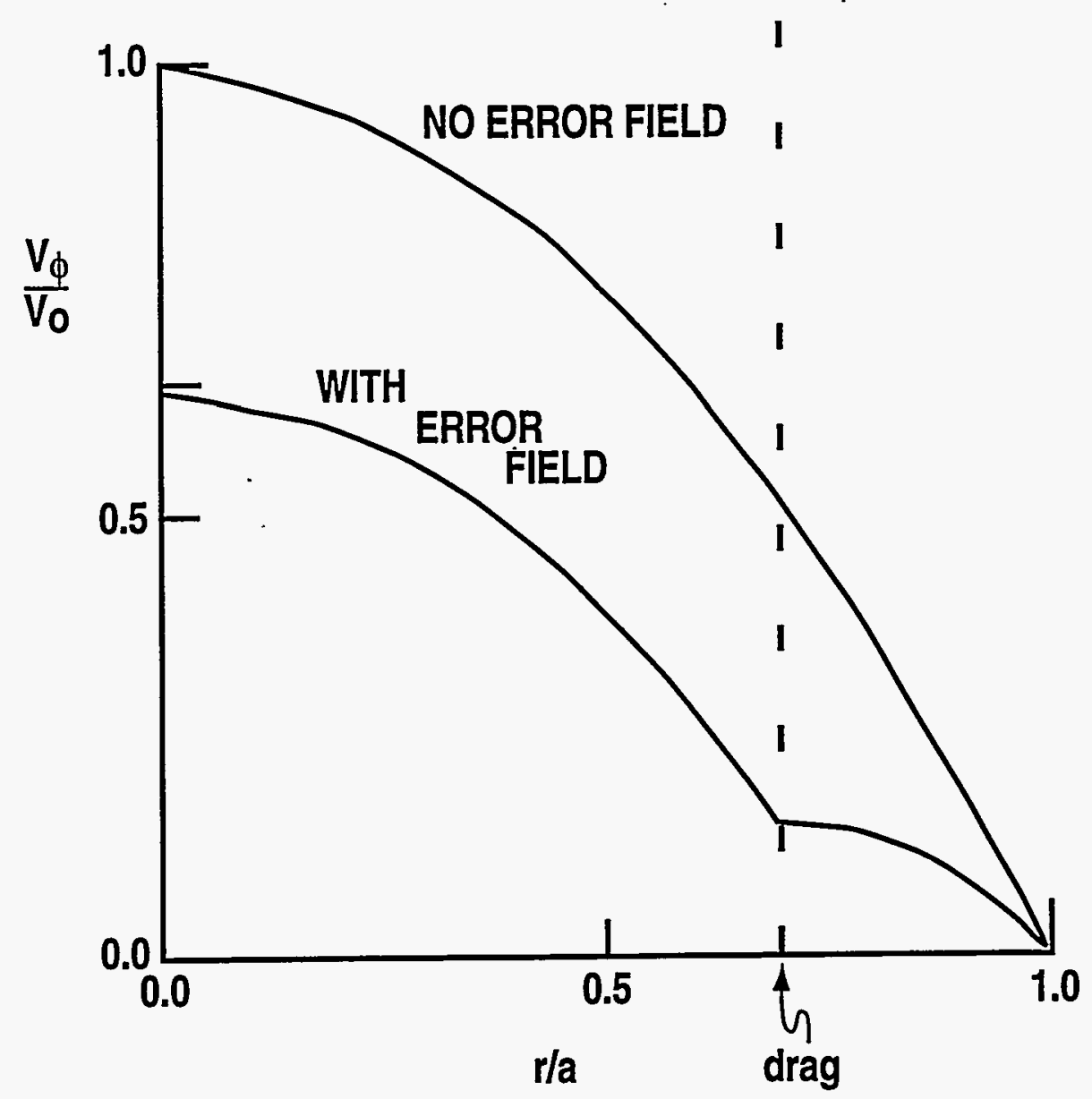

Fig. 1. Model radial profiles of plasma rotation with and without error field delta function drag at singular surface $r_{m n} / a=0.7$ for $T_{\phi m n} /\left[2 \pi^{2} R a^{2} d T_{\phi} / d V\right]=0.5$.

\subsection{SINGLE $m / n$ MODE, 1-D MODEL OF RESONANT ERROR FIELD MODE LOCKING}

The momentum balance with a drive $T_{\phi}$ and a characteristic angular rotation $\omega_{\phi}$ is

$$
0 \approx T_{\phi}-\bar{n} m_{\mathrm{i}} 2 \pi R \pi a^{2}\left(\frac{v_{\perp}}{a^{2}} \frac{\omega_{\phi} R}{2}\right) R-\frac{C B_{r m n}^{2}}{\omega \tau_{\text {rec }}},
$$

with $C=\left(4 \pi^{2} R r_{m n}^{2} / q \mu_{0}\right) 2 \ln \left(a / r_{m n}\right)$ approximating the right geometric factor for the location of the error field drag. For zero error field, $B_{r m n}=0, \omega_{\phi} \equiv \omega_{0}=T_{\phi} / \pi^{2} \bar{n} m_{\mathrm{i}} v_{\perp} R^{3}$ is the unperturbed angular rotation. As $B_{r m n}^{2}$ is increased, $\omega$ decreases and this allows more reconnection ( $\omega \tau_{\text {rec }}$ is smaller) increasing the normalized drag, etc. A critical condition is reached for mode locking. The angular rotation with $B_{r m n}^{2}$ is $[7,18]$

$$
\frac{\omega_{\phi}}{\omega_{0}}=\frac{1}{2}+\frac{1}{2}\left(1-\frac{4 C B_{r m n}^{2}}{\omega_{0} \tau_{\mathrm{rec}} T_{\phi}}\right)^{1 / 2} .
$$


For $\omega / \omega_{0} \leq 1 / 2$, there are no solutions and a discontinuous jump is made to $\omega / \omega_{0} \approx 0$ and $\omega \tau_{\text {rec }}<1$, i.e., locking. The critical error field relative to $B_{T}$ for $\omega / \omega_{0}=1 / 2$ can be written as

$$
\frac{B_{r m n}}{B_{\mathrm{T}}}=\frac{\omega_{0} \tau_{\mathrm{A}}}{4}\left(\frac{\tau_{\mathrm{rec}}}{\tau_{\mathrm{V}}}\right)^{1 / 2} \frac{q^{1 / 2}}{\left[2 \ln \left(a / r_{m n}\right)\right]^{1 / 2}}
$$

Equation (7) gives the basic scaling for locking. Note that since $\tau_{\mathrm{A}} \sim \bar{n}^{1 / 2}, B_{r m n} / B_{\mathrm{T}}$ scales as $\omega_{0} \bar{n}^{1 / 2}$ indicating a faster unperturbed rotation is less sensitive to error field locked modes ( $\omega \tau_{\text {rec }}$ is bigger, $T_{\phi m n} / B_{r m}^{2}$ is smaller) and a higher density is less sensitive (for fixed $\omega_{0}$ ) as the mass the error field torque must viscously slow is greater. The behavior of $\omega / \omega_{0}$ and $w / w_{\text {lock }}$ as $B_{r m n}$ is increased from 0 to $B_{\text {crit }}$ are shown in Fig. 2. For ITER ohmic plasmas taking $m / n=2 / 1, \omega_{0} / 2 \pi=130 \mathrm{~Hz}, \tau_{\mathrm{A}}=0.4 \mu \mathrm{sec}, \tau_{\text {rec }}=0.25 \mathrm{sec}$, $\tau_{\mathrm{v}}=\tau_{\mathrm{E}}=1.7 \mathrm{sec}, a / r_{m n}=1.4$, one gets $B_{r 21} / B_{\mathrm{T}} \approx 5 \times 10^{-5}$ which will be more rigorously treated in Section 4.

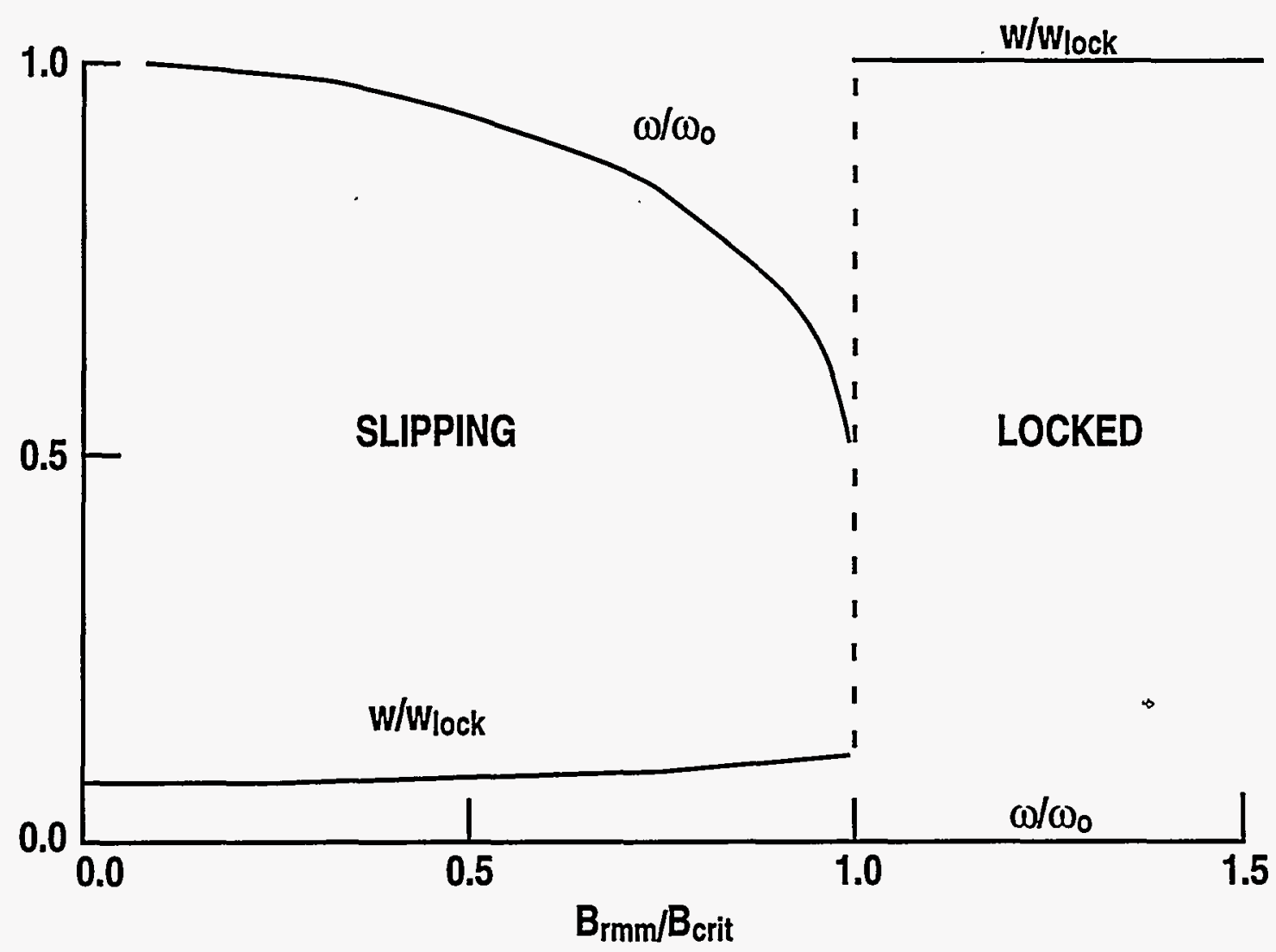

Fig. 2. Model for relative plasma rotation $\omega / \omega_{0}$ and island width $w / w_{\text {lock }}$ versus $m / n$ resonant static radial error field normalized to critical value for mode locking. 
The Jensen et al., 1993 skin layer physics gave a discontinuous locking at $\omega / \omega_{0}=2 / 5$ and the nonlinear resistivity model (Jensen et al., 1996) gives a nest of curves, some discontinuous - some not. Experimentally, it is difficult to detect the difference as $\omega / \omega_{0} \approx 1 / 2$ at locking. As an aside, note that while the original Fitzpatrick and Hender theory of 1991 was quite successful, it made assumptions on a critical condition at $\omega \tau_{\text {rec }} \approx 1$ (not $\omega \tau_{\text {rec }} \gg 1$ ) which maximized $T_{\phi m n}$ and involved $\Delta^{\prime}$ as a parameter [13]. Later, it was recognized that the slipping torque along with $\left(\omega \tau_{\text {rec }}\right)^{2} \gg 1$ could describe the critical condition through Eq. (6) which does not explicitly involve $\Delta^{\prime}$ [7]. 



\section{MULTIMODE $\mathrm{m} / \mathrm{n}$ LOCKING}

Locking can, in principle, occur by any resonant mode $m / n$. However, modes such as $1 / 1$ or $3 / 2$ that resonate near the axis have little torque as surface area is small, rotation is high, and the plasma is hotter for a larger $\tau_{\text {rec }}$ [Eq. (2)]. The modes produced by external sources fall off as $r^{m-1}$ going in radially to the surfaces, thus low $m$ is likely to have a stronger $B_{r m n}^{2}$. Experimentally, $m / n=2 / 1$ is most troublesome. A further complication arises through multimode effects, which can be significant even for low beta, ohmic plasmas in at least two ways.

The first way is through $m \pm 1, n$ sideband toroidal coupling to the $m, n$ surface. The effective 2,1 static error has two sources: (1) the external 2,1 component $B_{r 21}$, and (2) the plasma-induced 2,1 from external coil 1,1 and 3,1 errors. If there are $q=1$ and 3 surfaces, there will be plasma rotation eddy currents to oppose reconnection, which nearly cancel the 1,1 and 3,1 error fields. The $B_{r 2 \pm 1,1}$ fields induced at $q=2 \pm 1$ will have sidebands at $q=2$ of order $r_{21} / R$.

Thus, the total effective 2,1 component $\Sigma B_{r 21}$ taking into account the $r^{m-1}$ falloff of $B_{r m n}$ inside the source and $r^{(m+1)}$ outside is,

$$
\sum B_{r 21} \approx B_{r 21}+B_{r 11}\left(\frac{r_{11}}{r_{21}}\right)^{2} \frac{r_{21}}{R}+B_{r 31}\left(\frac{r_{21}}{r_{31}}\right)^{2} \frac{r_{21}}{R} .
$$

Of course, the phases must be taken into account properly which is not done for simplicity in Eq. (8). If only $B_{r 21}$ were critical for locking, nulling the external $B_{r 21}$ alone could leave a residual effective $B_{r 21}$ from the external $B_{r 11}$ and $B_{r 31}$ This would shift any 2,1 correcting coil scheme to a somewhat different optimum correction and for typical aspect ratio of $a / R \approx 1 / 3$ is a very small effect.

The second way of multimode locking is through the effect of resonant static error drag on multiple surfaces. Rotation reduction by 1,1 and/or 3,1 modes can lower $\omega_{\phi}$ viscously at $q=2$ for example, allowing a smaller $B_{r 21}$ to produce locking. The " $0-\mathrm{D}$ " momentum balance can be rewritten as

$$
0=T_{\phi}-\bar{n} m_{\mathrm{i}} 2 \pi R \pi a^{2}\left(\frac{v_{\perp}}{a^{2}} \frac{\omega_{\phi} R}{2}\right) R-\sum_{m} \sum_{n} \frac{C_{m n} B_{r m n}^{2}}{\omega_{\phi} \tau_{\mathrm{rec}, m n}}
$$

where 


$$
\begin{aligned}
C_{m n} & \approx \frac{4 \pi^{2} R r_{m n}^{2}}{q \mu_{0}}\left[2 \ln \left(a / r_{m n}\right)\right] \text { for } r_{21}<r_{m n} \\
& \approx \frac{4 \pi^{2} R r_{m n}^{2}}{q \mu_{0}}\left[2 \ln \left(a / r_{21}\right)\right] \text { for } r_{21}>r_{m n}
\end{aligned}
$$

with $\omega_{\phi}$ the 2,1 surface rotation.

The new critical condition for 2,1 locking is:

$$
\left[\sum_{m} \sum_{n} \frac{\left(C_{m n} / C_{21}\right)\left(B_{r m n} / B_{\mathrm{T}}\right)^{2}}{\tau_{\mathrm{rec}, m n} / \tau_{\mathrm{rec}, 21}}\right]^{1 / 2}=\frac{\omega_{0} \tau_{A}}{4}\left(\frac{\tau_{r e c}}{\tau_{V}}\right)^{1 / 2} \frac{q^{1 / 2}}{\left[2 \ln \left(a / r_{21}\right)\right]^{1 / 2}},
$$

with the RHS evaluated at $q=2 / 1$ and the LHS evaluated over all significant modes, $1 / 1$, $2 / 1,3 / 1$ for example if not also $3 / 2,5 / 2$, etc. The LHS of Eq. 10) can be rewritten as $\left[\Sigma_{m} \Sigma_{n} w_{m n}\left(B_{r m n} / B_{\mathrm{T}}\right)^{2}\right]^{1 / 2}$ with $w_{m n}$ mode weights. If $v_{\perp}$ is flat, $r_{11} / a=0.3, r_{21} / a=0.7$, $r_{31} / a=0.9, T_{\mathrm{e}}$ is parabolic to $3 / 2, n_{\mathrm{e}}$ is parabolic, and $\tau_{\mathrm{rec}} \approx \tau_{\mathrm{R}}^{5 / 6} \tau_{\mathrm{A}}^{1 / 3} / \tau_{\mathrm{v}}^{1 / 6}$ as suggested by Fitzpatrick, one gets $w_{11}: w_{21}: w_{31}=0.6: 1.0: 1.5$. For DIII-D ohmic discharges at $q_{95} \approx 3.2$, the empirically determined weights are found to be $0.2,1.0,0.8[20,21] . n=2$ resonant errors are twice as high $m$ for the same $q$ and thus should decay away faster as $B_{r m n} \sim r^{m-1}$. COMPASS-C with a nearly pure $m / n=4 / 2$ winding could produce locked modes but the source current had to be high [22].

Finally, elongation can couple $m \pm 2, n$ to $m, n$ but this is a weaker effect than toroidal coupling. 


\section{ITER MULTI-MODE RESONANT STATIC ERROR LIMITS}

\subsection{OHMIC, LOW DENSITY}

The ohmic target density on ITER must be low to allow $\mathrm{H}$-mode at low supplementary heating power. Thus for $q_{95} \gtrsim 3, G=\bar{n}_{14} \pi a^{2} / I_{\mathrm{p}} \approx 0.2$ at $21 \mathrm{MA}, 2.8 \mathrm{~m}$, and $\bar{n} \approx 0.17 \times 10^{14} \mathrm{~cm}^{-3}$, the natural rotation will be that of the electron drift $\omega_{\mathrm{De}} / 2 \pi$ $\approx T_{\mathrm{e} 0} / B_{\mathrm{T}} a^{2}$ [5]. For low density, $\tau_{\mathrm{E}}$ is Pfeiffer/Waltz (neo-Alcator)

$$
\tau_{\mathrm{E}} \approx 9.5 \times 10^{-20} \bar{n}^{0.90} a^{0.90} R_{0}^{1.63},
$$

or about $1.7 \mathrm{sec}, T_{\mathrm{e} 0} \approx 4.2 \times 10^{6}\left(\tau_{\mathrm{E}} / \bar{n}\right)^{2 / 5}\left(I_{\mathrm{p}} / \pi a\right)^{4 / 5}$ from Spitzer or about $6 \mathrm{keV}$. Thus $\omega_{\mathrm{De}} / 2 \pi \approx 130 \mathrm{~Hz}$.

Matched experiments [9] for locking at $q \approx 3.5$ on COMPASS-C $(1.1 \mathrm{~T}, 0.56 \mathrm{~m})$ [22]. DIII-D $(1.3 \mathrm{~T}, 1.7 \mathrm{~m})$ [23], and JET $(3.1 \mathrm{~T}, 3.0 \mathrm{~m})$ [24] had $\omega_{\mathrm{De}} / 2 \pi \approx 14,1.6$, and $0.6 \mathrm{kHz}$, respectively, which is consistent with scaling to $130 \mathrm{kHz}$ for ITER at $5.7 \mathrm{~T}, 8.0 \mathrm{~m}$. The measured conditions for locking at $G \approx 0.2, q \approx 3.5$, ohmic, deuterium are given in Table I for both the $2 / 1$ mode only and for the weighted conditions using empirically determined weights from DIII-D. Extrapolation with $R$ gives a critical $B_{r 21} / B_{\mathrm{T}}=1.0 \times 10^{-5}$ for ITER or a weighted $B_{\text {reff }, 1} / B_{\mathrm{T}}=1.9 \times 10^{-5}$. (As $\tau_{\mathrm{E}} \sim \bar{n}, T_{\mathrm{e} 0} \sim\left(I_{\mathrm{p}} / a^{2}\right)^{4 / 5}, \omega_{\mathrm{De}} \sim T_{\mathrm{e} 0} / B_{\mathrm{T}} a^{2}$ $\sim R^{-9 / 5} B^{-1 / 5}$ decreases rapidly with $R$ and hardly at all with $B$.) A plot of critical error field versus $R$ for both kinds of criticality is given in Fig. 3. This is consistent with Eqs. (7) and (10) for $\omega_{\mathrm{De}} \sim R^{-9 / 5}$ as the dominant variable and experimentally pins down a number of approximate factors.

In this scaling, the definition of $B_{r m n}$ in each case is: (1) ohmic plasma, $q_{95}$ or $q_{\lim }=3.5$, (2) $m / n$ components decomposed on the $q=2$ surface, (3) the "straight field line" coordinate used is that coordinate $\theta^{*} \simeq \theta_{\text {cyl }}+\varepsilon \lambda \sin \theta_{\text {cyl }}$ where $\varepsilon=r / R$, $\lambda=\beta_{\mathrm{p}}+\ell_{\mathrm{j}} / 2-1$ or better yet found by magnetic field line tracing, (4) $B_{r m n} \equiv B_{\perp m n}$ perpendicular to $q=2$ surface, and (5) helical Fourier analysis of resonant hand mode of interest $\cos \left(n \phi-m \theta^{*}\right)$ not $\cos n \phi \cos m \theta^{*}$, etc. Based on both theory/modeling and COMPASS-C, DIII-D, and JET experiments, a simultaneous ohmic limit for critical error field components is given in Table $\Pi$. (This is a best fit of the data versus $R$.) This adds up to a weighted $1.9 \times 10^{-5}$ and includes the perhaps most dangerous $n=2$ mode (whose $r^{3}$ fall off may make it easy to avoid). 
Table I: Scaling to ITER, Multi-mode Experiments, Simultaneous

$$
\left(G \approx 0.2, q \approx 3.5 \text {, ohmic, D2, } w_{11} \equiv 0.2, w_{21} \equiv 1.0, w_{31} \equiv 0.8\right. \text { ) }
$$

\begin{tabular}{lccccc}
\hline Device & $R(\mathrm{~m})$ & $B_{r 11} / B_{\mathrm{T}}$ & $B_{r 21} / B_{\mathrm{T}}$ & $B_{r 31} / B_{\mathrm{T}}$ & $\sqrt{\Sigma w_{m 1}\left(B_{r m 1} / B_{\mathrm{T}}\right)^{2}}$ \\
\hline COMPASS-C & 0.56 & - & $1.6 \times 10^{-3}$ & - & $1.6 \times 10^{-3}$ \\
DIII-D & 1.67 & $2.8 \times 10^{-4}$ & $2.2 \times 10^{-4}$ & $2.5 \times 10^{-4}$ & $3.3 \times 10^{-4}$ \\
JET & 3.0 & $8.4 \times 10^{-5}$ & $6.4 \times 10^{-5}$ & $4.3 \times 10^{-5}$ & $8.8 \times 10^{-5}$ \\
& & & $\uparrow$ & & $\uparrow$ \\
& & & best fit & & best fit \\
& & & $B_{r 21} / B_{\mathrm{T}}=5.4 \times 10^{-4} R^{-1.90}$ & $B_{\text {reff }} / B_{\mathrm{T}}=5.4 \times 10^{-4} R^{-1.69}$ \\
& & $\left(r^{2}=-0.999\right)$ & & $\left(r^{2}=-0.993\right)$ \\
ITER & & & $\downarrow$ & & $\downarrow$ \\
& & & $1.0 \times 10^{-5}$ & & $1.9 \times 10^{-5}$ \\
\hline
\end{tabular}

\subsection{NEUTRAL BEAM ROTATION DRIVE}

The critical error field scales as $B_{r 21} / B_{\mathrm{T}} \sim \omega_{0} \bar{n}^{1 / 2}$. Increasing density is less effective in obviating tight error limits than increasing rotation and is incompatible with a low $\mathrm{L} \rightarrow \mathrm{H}$ transition power. Increasing $\omega_{0}$ in an ohmic plasma requires changing the electron diamagnetic drift as $\omega_{0} / 2 \pi \approx T_{\mathrm{e} 0} / B a^{2}$. However for $\tau_{\mathrm{E}} \sim \bar{n}, T_{\mathrm{e} 0} \sim\left(I_{\mathrm{p}} / a^{2}\right)^{4 / 5}$ and $\omega_{0}$ $\sim R^{-9 / 5} B^{-1 / 5}$ cannot be modified once $R$ and $B$ are chosen.

Co-injected neutral beams in DII-D L-mode plasmas are successful in increasing the rotation and raising the critical error field [25]. However co-injection opposes the electron drift and has two problems: (1) $\left|\omega_{0}\right| \approx \mid\left(\omega_{D e}-\omega_{\text {fluid }}\right) l$, so is inefficient unless $\left|\omega_{\text {fluid }}\right| \gg\left|\omega_{\text {De }}\right|$ and (2) $\omega_{0} \approx 0$ is transiently passed through during which the plasma is very sensitive to locking. (In practice if $\omega_{0} \approx 0$ is passed through rapidly in a time less than the locking time, the plasma survives.) Counter beams would thus be both more efficient, $\left|\omega_{0}\right| \approx\left|\omega_{\text {De }}+\omega_{\text {fluid }}\right|$, and not pass $\omega_{0}$ through zero.

Ideally, the beam should be as tangential as possible $\left(\cos \psi_{\mathrm{B}} \approx 1\right)$ and of as low voltage $V_{\mathrm{B}}$ as possible, compatible with penetration, to provide the highest angular momentum transfer per unit beam energy. With $\omega_{\mathrm{De}} / 2 \pi \approx 130 \mathrm{~Hz}$ for an ohmic plasma at $G \approx 0.2$, assuming the momentum and energy confinement times are equal, $\tau_{\mathrm{M}} \approx \tau_{\mathrm{E}}$, and $\tau_{\mathrm{E}} \approx \tau_{\mathrm{L}}$, the L-mode confinement [25]

$$
\tau_{\mathrm{L}} \approx 0.5 \times 0.106 * P_{\text {loss }}^{-0.46}(\mathrm{MW}) I_{\mathrm{p}}^{1.03}(\mathrm{MA}) R^{1.48}(\mathrm{~m})
$$




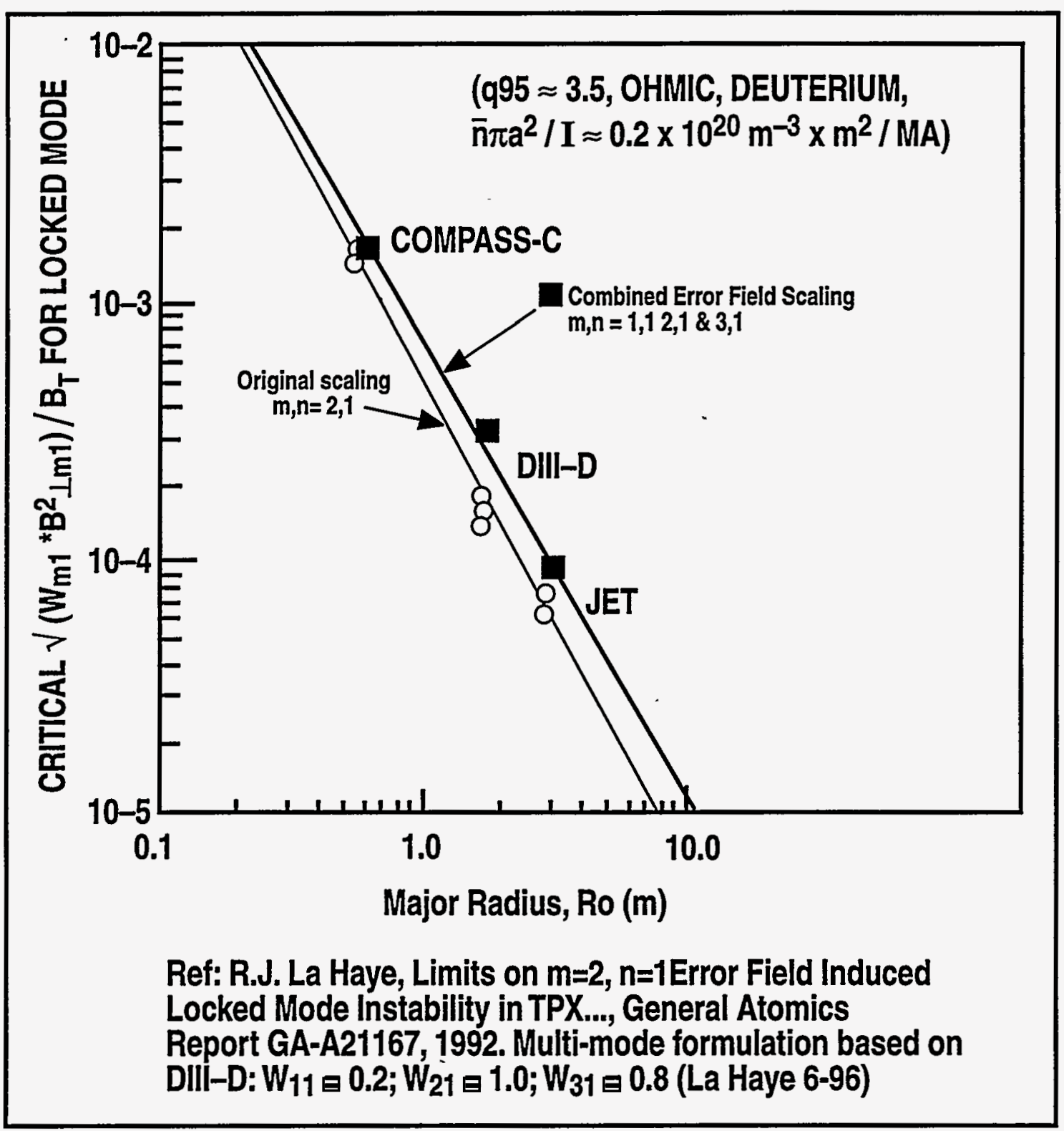

Fig. 3. Critical weighted relative error field versus major radius for COMPASS-C, DIII-D, and JET extrapolated to ITER. (Ohmic, $q_{95} \approx 3.5$, deuterium, $G=\bar{n} \pi a^{2} / I \approx 0.2$.) The lines are best fit with components given in Table I.

with $P_{\text {loss }}=P_{\Omega}+P_{\mathrm{B}}$, one gets fluid rotation at $q=2$

$$
\omega / 2 \pi \approx \frac{\mathrm{v}_{\mathrm{B}} P_{\mathrm{B}} \tau_{\mathrm{M}} \cos \psi_{\mathrm{B}}}{e V_{\mathrm{B}} \bar{n} 2 \pi R \kappa \pi a^{2} 2 \pi R},
$$

with $\mathrm{v}_{\mathrm{B}}=\left(\kappa_{\mathrm{B}} V_{\mathrm{B}} / m_{\mathrm{D}}\right)^{1 / 2}$ and elongation $\kappa$. Case $\mathrm{I}, P_{\mathrm{B}}=5 \mathrm{MW}$ at $1 \mathrm{MeV}$ at $45^{\circ}, P_{\Omega}$ $=12 \mathrm{MW}$ for $0.6 \mathrm{~V}$ at $21 \mathrm{MA}, P_{\text {loss }}=17 \mathrm{MW}^{\prime}$ for $\tau_{\mathrm{L}} \approx 8 \mathrm{sec}$, and $\omega / 2 \pi \approx 520 \mathrm{~Hz}$ or $\left(\omega+\omega_{\mathrm{De}}\right)$ a factor of 5 larger than $\omega_{\mathrm{De}}$. Case II, $P_{\mathrm{B}}=13 \mathrm{MW}, P_{\Omega}=12 \mathrm{MW}, P_{\text {loss }}$ $=25 \mathrm{MW}$ for $\tau_{\mathrm{L}} \approx 7 \mathrm{sec}$, and $\omega / 2 \pi \approx 1700$ for $\left(\omega+\omega_{\mathrm{De}}\right)$ a factor of 10 larger than $\omega_{\mathrm{De}} \cdot$ 


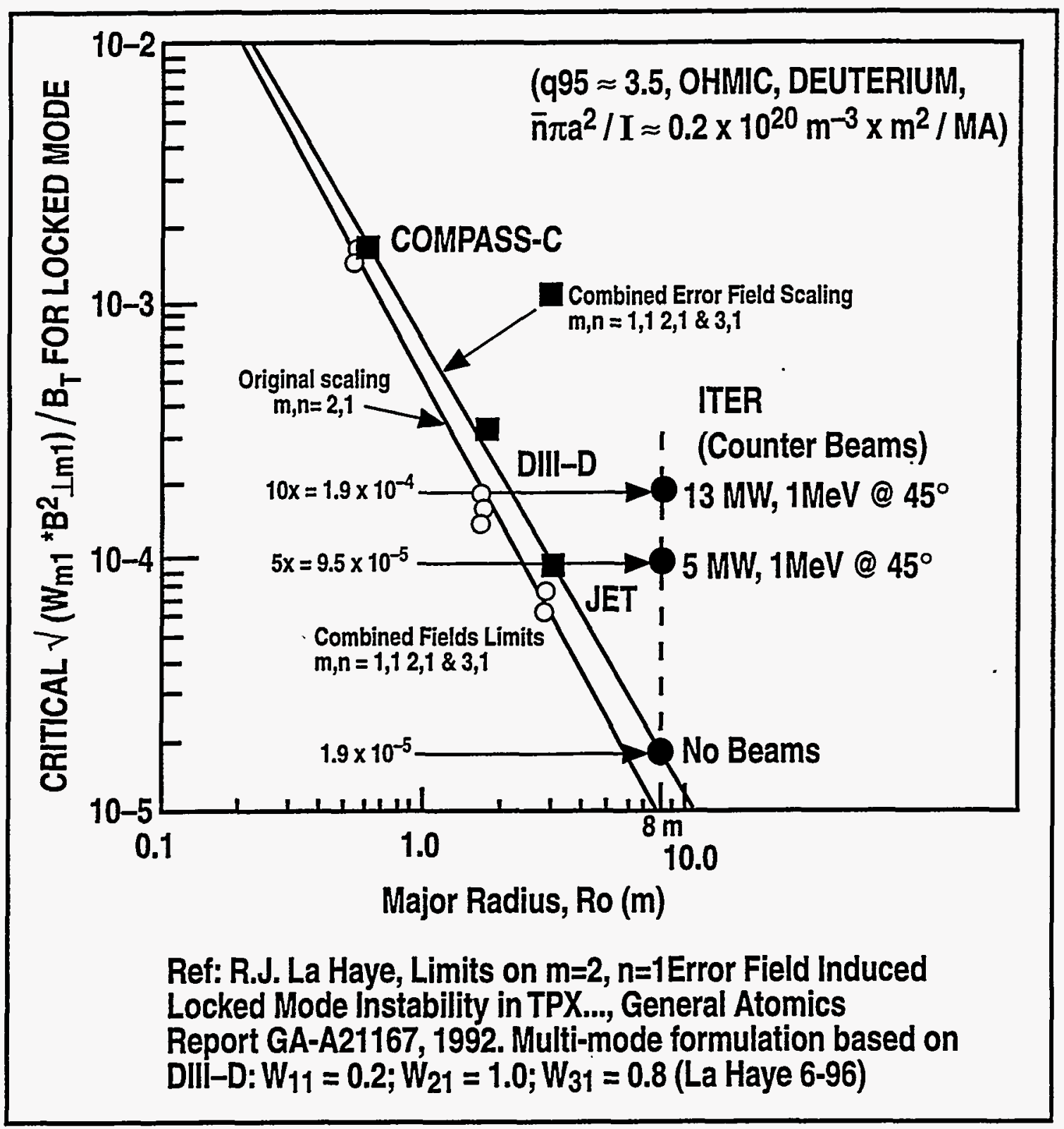

Fig. 4. Same as Fig. 3 but calculated effect of $1 \mathrm{MeV}$ counter-injected beams at $45^{\circ}$ included for ITER.

Either power is below the threshold to stay in $L$-mode at $G \approx 0.2\left(1.7 \times 10^{13} \mathrm{~cm}^{-3}\right)$ which requires $P_{\mathrm{B}}<0.025 \bar{n} B_{\mathrm{T}} S=33 \mathrm{MW}$ for $5.7 \mathrm{~T}$ and $S=1150 \mathrm{~m}^{2}$ surface area. Thus using Eqs. (7) or (10), the allowable error field before locking could be increased a factor of 5 (case I) or 10 (case II) in a low density L-mode target. This is shown in Fig. 4 . The scenario would be to apply beams in the startup phase when $q$ is higher and $q=2$ is deeper into the plasma (thus $r^{m-1}$ weaker error field) so as to arrive at an L-mode, low density current flattop with enough rotation to avoid locked modes. Of course beam fueling could increase the density above $G \approx 0.2$. Using co-beams would (if locking at $\omega+\omega_{\mathrm{De}} \approx 0$ is 
PHYSICS OF LOCKED MODES IN ITER: ERROR FIELD LIMITS, ROTATION FOR OBVIATION, AND MEASUREMENT OF FIELD ERRORS

Table II: Proposed Simultaneous ITER Error Field Limits

(ohmic weights $w_{11}=0.2, w_{21}=1.0, w_{31}=0.8$, and $w_{42}=0.5$ )

\begin{tabular}{ccc}
\hline$m$ & $n$ & $B_{r m n} / B_{\mathrm{T}}\left(10^{-5}\right)$ \\
\hline 1 & 1 & 2.2 \\
2 & 1 & 1.0 \\
3 & 1 & 1.1 \\
4 & 2 & 0.7 \\
\hline
\end{tabular}

avoided) decrease the factors from 5 and 10 to 3 and 8, respectively. The proposed simultaneous limits shown in Table II could thus be raised a factor of 3 to 10 depending on co or counter and the beam power (and angle and voltage) used. 



\section{ERROR FIELD MEASUREMENT AND COIL ALIGNMENT}

\subsection{INTRODUCTION}

It is not impossible to achieve single resonant mode relative error fields of $B_{r m n} / B_{\mathrm{T}}$ $\sim 1 \times 10^{-5}$. This was done in COMPASS $-\mathrm{C}$ by careful coil design and alignment of coils to minimize the magnetic field error on a precise array of pickup coils $[10,26]$. The COMPASS-C array is shown in Fig. 5 both as permanently installed and free standing. The array was iteratively moved to be concentric with the circular magnetic field line of the pulsed toroidal field TF coil (assumed perfect, i.e., errorless). Then each poloidal field PF coil was pulsed individually and the error fieids $B_{R}, B_{\phi}, B_{Z}$ at the array measured, followed by moving the PF coil iteratively until the error fields were "nulled" out. This leaves the PF coil fields axisymmetric with the TF coil field, as desired. (Any PF coil known irregularities can be calculated at the array and compensated for in the alignment.) Sub millimeter alignment was achieved for each coil. Since $R_{\mathrm{ITER}} / R_{\mathrm{COMPASS}} \approx 13$, a $0.5 \mathrm{~mm}$ alignment tolerance on COMPASS would be equivalent to $6.5 \mathrm{~mm}$ on ITER if the relative coil geometry was the same.

In DIII-D, error field measurement was done about four years after assembly when locked modes were recognized as a problem in operation. An array (see Fig. 6) similar to that of COMPASS-C was built on a rigid octagonal frame to sub $\mathrm{mm}$ accuracy and temporarily reassembled inside the DIII-D vacuum vessel [12]. Photoresist manufactured magnetic pickup coils were matched to produce a variation in effective pickup area of less than $6 \times 10^{-5}$. As in the smaller COMPASS-C device, the array was moved (in about 12 iterations) to be concentric with the assumed axisymmetric toroidal field. . $N=24$ ripple is not exhibited by an $N=8$ array of pickup coils.) The PF coils could not be realigned in DIII-D but mm irregularities in placement were measurable and used for calculation of the total $B_{r m n}$ for correction by the " $n=1$ " and "C" coils in DW-D.

\subsection{PROPOSED ITER MEASUREMENT AND ALIGNMENT STEPS}

(1) Construct, as in DIII-D, a temporary rigid frame of eight triplet ( $B_{R}, B_{Z}, B_{\phi}$ ) pickup coils by photoresist mat. The coils should have $\delta N A / N A<6 \times 10^{-5}$ matching, be positioned on a great circle of $R=8.0 \mathrm{~m}$ to $\Delta Z, \Delta R<1 \mathrm{~mm}$, and $\Delta \psi<0.02^{\circ}(0.6 \mathrm{~mm})$ orthogonality. Opposite coils are subtracted and then integrated to get $n=1$ field components. ( $n=2$ is also readily doable as in DIII-D [12].) The array is temporarily 


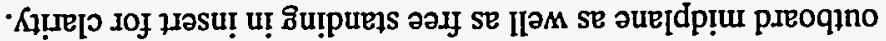

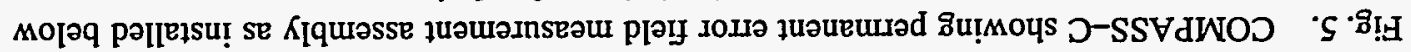
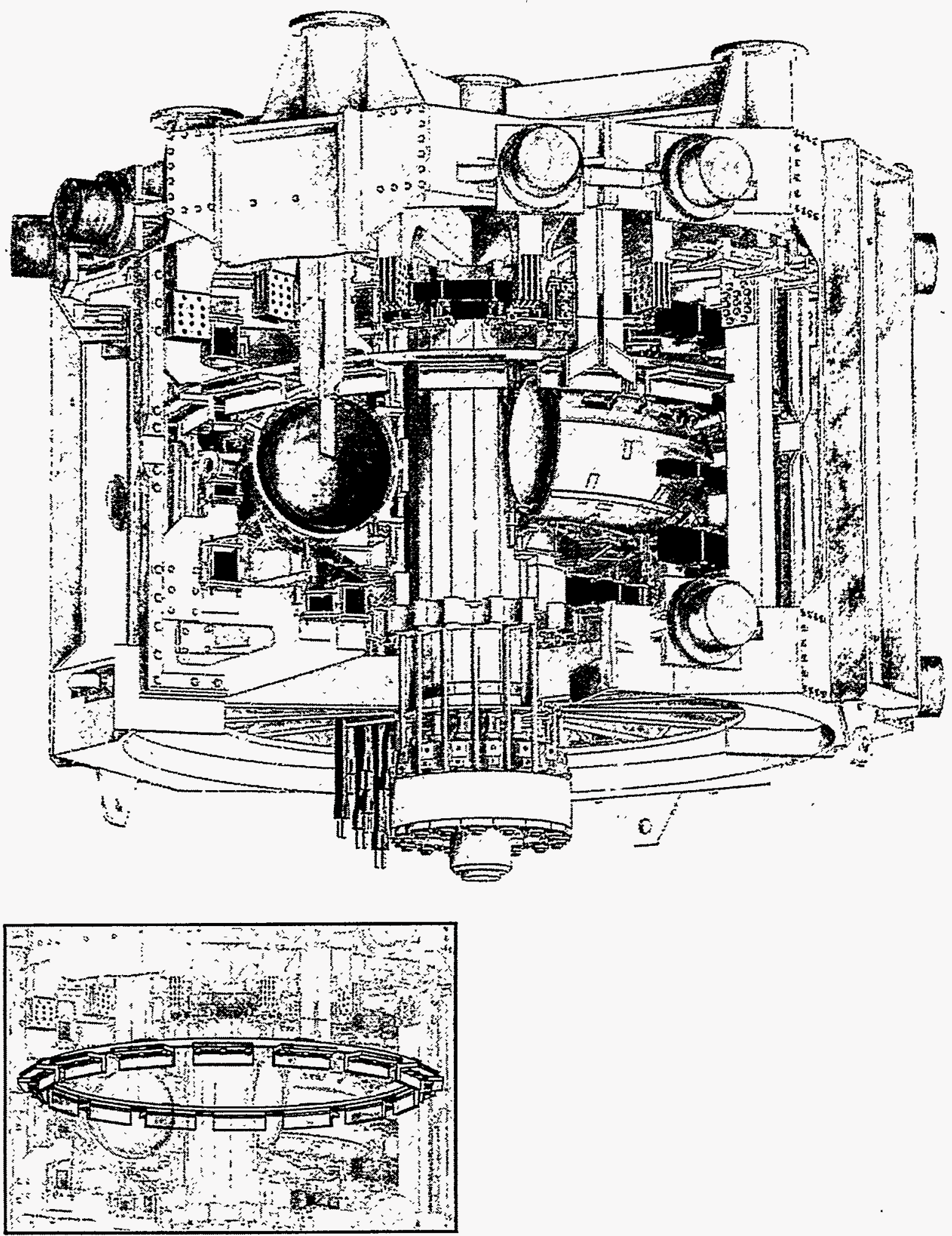


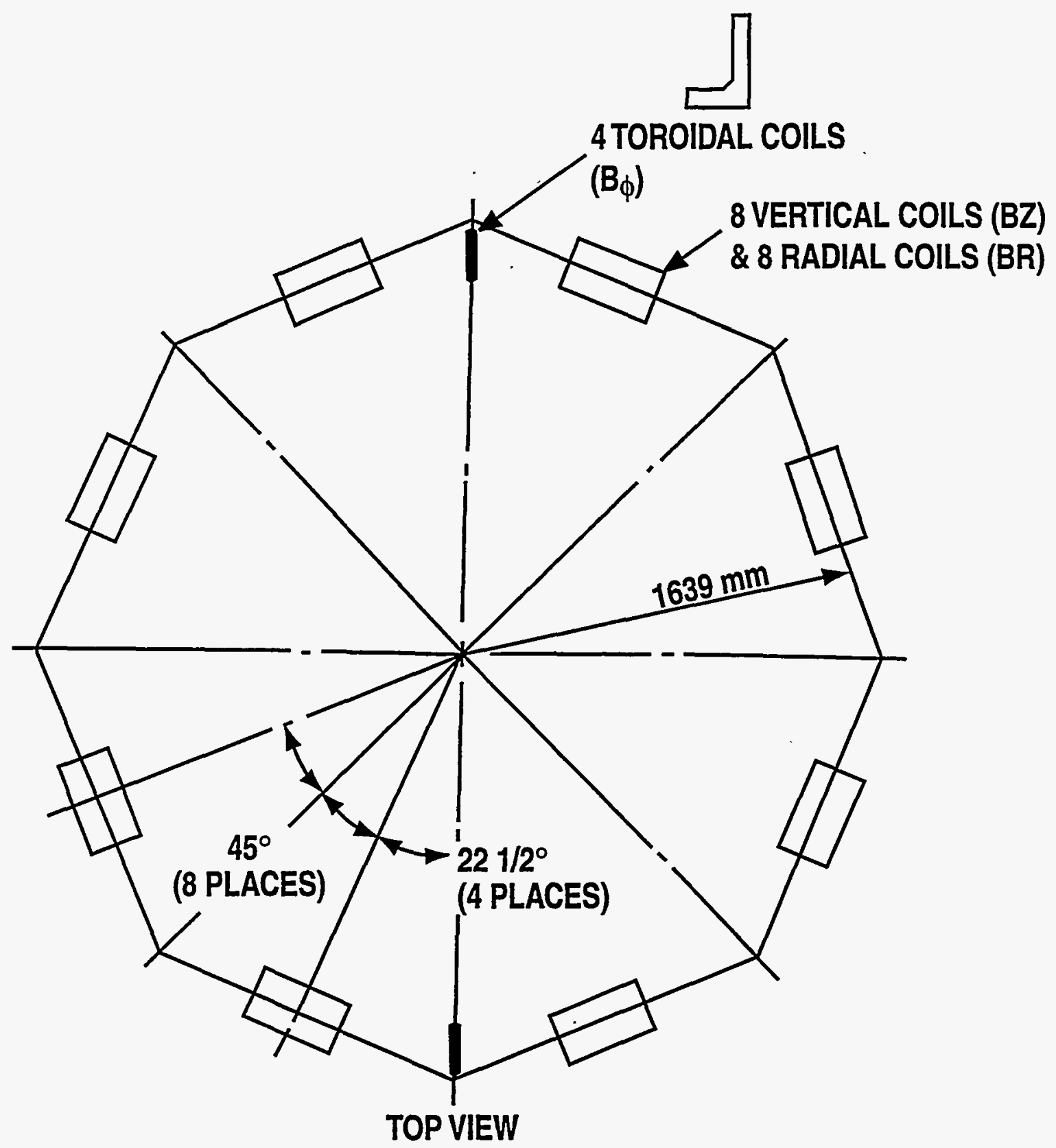

Fig. 6. DIII-D top view of pickup coil array used to temporarily measure error fields in center of vacuum vessel.

assembled at the nominal midplane, $Z=0, R=8 \mathrm{~m}$ inside ITER but does not depend critically on the nominal location.

(2) Assume the TF coil is "perfect," i.e., only $n=0$ and $n=20$ ripple fields with ripple negligible at $R=8 \mathrm{~m}$. Energize the TF coil. Measure $n=1$ pickups. The horizontal displacements from the array concentric to the toroidal field are $\Delta X, \Delta Y \approx 0.5\left(\Delta B_{\phi 1} / B_{\phi}\right) R$ from opposite pairs of $B_{\phi}$ pickups. If matching of photoresist coils is good to $6 \times 10^{-5}$, alignment can be made iteratively by moving the whole array to $\Delta X, \Delta Y$ 
$\leq 0.25 \mathrm{~mm}$ less than the alignment on the frame. The tilt of the array to the toroidal field is $R \Delta \phi \approx 0.5\left(\Delta B_{Z 1} / B_{\phi}\right) R \approx 0.25 \mathrm{~mm}\left(0.002^{\circ}\right)$ for coils matched to $6 \times 10^{-5}$, so alignment to the plane of the toroidal field can be made iteratively to $R \Delta \phi \lesssim 0.25 \mathrm{~mm}$.

Now if the TF coil has an irregularity such as $n=1$, the array will be aligned not to the $n=0$ toroidal field but slightly off the $n=0$ toroidal field. As modes decay as $r^{m-1}$ toward the axis, the dominant error will be of form

$$
\begin{aligned}
& B_{\phi 1} \approx-\frac{r}{R} B_{1} \sin (\phi-\theta), \\
& B_{R 1} \approx B_{1} \cos (\phi-\theta), \\
& B_{Z 1} \approx B_{1} \sin (\phi-\theta),
\end{aligned}
$$

Let $B_{1} / B_{\phi}=1 \times 10^{-4}$ or $B_{1}=12 \mathrm{G}$ at $B_{\phi}=6 \mathrm{~T}$. The error in horizontal $\Delta X, \Delta Y$ $\approx(a / R)\left(B_{1} / B_{\phi}\right) R=0.03 \mathrm{~mm}$ if $\mathrm{r} \leq a$. The error in tilt $R \Delta \phi \approx 2\left(B_{\mathrm{Zl}} / B_{\phi}\right) R \approx 1.5 \mathrm{~mm}$. Thus a mm systematic misalignment of the array is possible if the $n=1$ total TF coil irregularity is not negligible. This can be determined as the iterative procedure is followed in later steps, to be discussed.

(3) Now with the array "concentric" to the assumed "perfect" toroidal field circle energize each PF coil one at a time, measuring the $n=1$ error fields. ( $n=2$ can also be done.) Ideally, the COMPASS-C procedure would be followed to iteratively move each coil to null the error field. If not, the DIII-D procedure is to compute the misalignment for use as a basis for error correction. Consider ITER poloidal field coil PF4 for example at $R=15.4 \mathrm{~m}, Z=-2.1 \mathrm{~m}, I_{\mathrm{c}}=11.2 \mathrm{MA}$-turns. At field point $Z=0, R=8.0 \mathrm{~m}$, $B_{R}=799 \mathrm{G}, B_{Z}=5447 \mathrm{G}, B_{\phi} \equiv 0$ with $\Delta B_{R} / \Delta R=0.235 \mathrm{G} / \mathrm{mm}, \Delta B_{Z} / \Delta R=0.310 \mathrm{G} / \mathrm{mm}$, $\Delta B_{R} / \Delta Z=0.310 \mathrm{G} / \mathrm{mm}$, and $\Delta B_{Z} / \Delta Z=-0.315 \mathrm{G} / \mathrm{mm}$. Any horizontal PF4 shift $\Delta_{\mathrm{H}}$ makes an $n=1$ error field as does any PF4 tilt $\Delta_{\mathrm{T}}$ from the plane of the toroidal field [8]. One calculates

$$
\begin{aligned}
& B_{R 1}^{(G)}=-0.235 \Delta_{\mathrm{H}}-0.550 \Delta_{\mathrm{T}}, \\
& B_{\mathrm{zl}}^{(G)}=0.310 \Delta_{\mathrm{H}}+0.260 \Delta_{\mathrm{T}},
\end{aligned}
$$

with $\Delta_{\mathrm{H}}, \Delta_{\mathrm{T}}$ in millimeters at the PF4 coil $R_{\mathrm{c}}, Z_{\mathrm{c}}$. There are four unknowns, the amplitude and the phase of $\Delta_{\mathrm{H}}$ and $\Delta_{\mathrm{T}}$, and four measurements, the amplitude and the phase of $B_{\mathrm{Z1}}$ and $B_{R 1}$. Typically a $\Delta_{\mathrm{H}}$ of $1 \mathrm{~mm}$ would give $\left|B_{R 1} / B_{R}\right| \approx 0.235 / 799 \mathrm{G} \approx 3 \times 10^{-4}$ and $\left|B_{Z 1} / B_{Z}\right| \approx 0.310 / 5419 \approx 6 \times 10^{-5}$ which are within the $\leqslant 6 \times 10^{-5}$ coil matching and the $\mathrm{mm}$ alignment to the toroidal field. Thus measuring an irregularity of order $1 \mathrm{~mm}$ in positioning/alignment of $\mathrm{PF}$ coils is possible. Any systematic misalignment of the array to 
the toroidal field "circle" can be readily checked for in the $\Delta_{\mathrm{H}}, \Delta_{\mathrm{T}}$ values of all PF-coils. Note that Gribov et al. calculate for a $6 \mathrm{~mm}$ horizontal shift at 11.2 MA-turns of PF4 only, $B_{r 21} / B_{\mathrm{T}} \approx 1 \times 10^{-5}$ at $q=2$ and for a $0.016^{\circ}$ tilt ( $4 \mathrm{~mm}$ at coil) only, $B_{r 21} / B_{\mathrm{T}} \approx 1 \times 10^{-5}$ at the hypothetical $q=2$ surface. These shifts/tilts are readily measurable. Of course the total $B_{r m n} / B_{\mathrm{T}}$ is the phase quadrature addition of all sources/coils.

(4) The PF4 only is an ideal curved vertical field without up/down symmetry for checking the total TF coil $n=1$ or $n=2$ error fields. Assuming PF4 has been realigned ( $a$ la COMPASS-C) or not (à la DIII-D) but is assumed perfectly axisymmetric, energize PF4 only and realign the pickup array to null $n=1$ as in step 2 noting displacements from alignment in step 2 to the toroidal field. Now the array is nominally concentric to the circle/plane of the assumed $n=0$ only poloidal field of PF4 at $R=8 \mathrm{~m}, Z=0$.

The TF coil only is energized and $n=1$ (2) error fields measured. Ideally the TF coil would be adjusted to null these errors but as the $N=20$ coil tilts, shifts, etc., possibilities include very many degrees of freedom, it may only be possible to document this error, not to fix it. A check of systematic error in array alignment in (2) could also be done from this measurement. (In addition, the magnetically aligned frame was useful in DIII-D for measuring vessel/limiter alignment points.) 

PHYSICS OF LOCKED MODES IN ITER: ERROR FIELD LIMITS, ROTATION FOR OBVIATION, AND MEASUREMENT OF FIELD ERRORS

\section{ACKNOWLEDGMENT}

This is a report of work supported by the U.S. Department of Energy under Contract No. DE-AC03-89ER51114. 


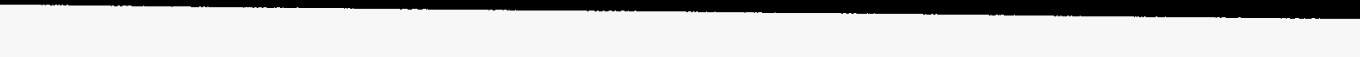




\section{REFERENCES}

[1] R.J. La Haye, General Atomics Report GA-C21120, October 1992.

[2] T.C. Hender et al., AEA Fus 230, March 1993.

[3] Y. Gribov et al., 3rd Plasma Control Task Force Meeting, ITER Naka JWS, April 1996.

[4] M.F.F. Nave and J.A. Wesson, Nucl. Fusion 30, 2575 (1990).

[5] T.H Jensen et al., Phys. Fluids B 3, 1650 (1991).

[6] T.H. Jensen et al., Phys. Fluids B 5, 1239 (1993).

[7] R. Fitzpatrick, Nucl. Fusion 33, 1049 (1993).

[8] O.A. Hurricane et al., Phys. Plasmas 2, 1976 (1995).

[9] R.J. La Haye et al., Phys. Fluids B 4, 2098 (1992).

[10] R.J. Crossland et al., Proceedings of the Symposium on Fusion Technology (SOFT) Conference, London 1990.

[11] J.D. Hanson, Nucl. Fusion 34, 441 (1994).

[12] R.J. La Haye and J.T. Scoville, Rev. Sci. Instrum. 62, 2146 (1991).

[13] G. Bateman, "MHD Instabilities" (MTT Press, 1978) p. 197.

[14] J.K. Lee et al., Nucl. Fusion 23, 63 (1983).

[15] T.H. Jensen et al., Phys. Plasmas 3, 1524 (1996).

[16] X. Wang and A. Bhattacharjee, to be published in Physics of Plasmas (1996).

[17] H. Zohm et al., Europhys. Lett. 11, 745 (1990).

[18] M. Yokoyama, J.D. Callen, and C.C. Hegna, University of Wisconsin Madison Report UW-CPTC95-4, November 1995.

[19] R. Fitzpatrick and T.C. Hender, Phys. Fluids B 3, 644 (1991).

[20] J.T. Scoville and R.J. La Haye, Bull. Am. Phys. Soc. 40, 1788 (1995).

[21] J.T. Scoville and R.J. La Haye, Report D3DPM No. 9604, August 1996.

[22] A.W. Morris et al., Phys. Fluids B 4, 413 (1992).

[23] J.T. Scoville and R.J. La Haye, Nucl. Fusion 31, 875 (1991).

[24] G.M. Fishpool and P.S. Haynes, Nucl. Fusion 34, 109 (1994).

[25] R.J. LaHaye, A.W. Hyatt, and J.T. Scoville, Nucl. Fusion 32, 2119 (1992).

[26] A.W. Morris and T.N. Todd, Culham Report CSC(88)3. 